Revue de l'Institut des langues et cultures

d'Europe, Amérique, Afrique, Asie et Australie

$23 \mid 2015$

Le national-socialisme dans son cinéma

\title{
Ewiger Wald et la figure tutélaire de Richard Wagner
}

Art total et instrumentalisation de la musique sous le nazisme

Ewiger Wald: Ein Kulturfilm im Zeichen Richard Wagners.

Kunst und weltanschauliche Instrumentalisierung der Musik im Dritten Reich

\section{Rémy Sanvoisin}

\section{OpenEdition}

Journals

Édition électronique

URL : http://journals.openedition.org/ilcea/3345

DOI : $10.4000 /$ ilcea.3345

ISSN : 2101-0609

Éditeur

UGA Éditions/Université Grenoble Alpes

Édition imprimée

ISBN : 978-2-84310-305-6

ISSN : $1639-6073$

\section{Référence électronique}

Rémy Sanvoisin, «Ewiger Wald et la figure tutélaire de Richard Wagner », ILCEA [En ligne], 23 | 2015,

mis en ligne le 09 juillet 2015, consulté le 22 avril 2019. URL : http://journals.openedition.org/ ilcea/3345 ; DOl : 10.4000/ilcea.3345

Ce document a été généré automatiquement le 22 avril 2019

(C) ILCEA 


\title{
Ewiger Wald et la figure tutélaire de Richard Wagner
}

\author{
Art total et instrumentalisation de la musique sous le nazisme \\ Ewiger Wald: Ein Kulturfilm im Zeichen Richard Wagners. \\ Kunst und weltanschauliche Instrumentalisierung der Musik im Dritten Reich
}

Rémy Sanvoisin

1 L'art constitue pour le nazisme l'un des principaux piliers de l'identité allemande. En premier lieu, c'est par la musique que le Parti national-socialiste cherche à promouvoir le passé artistique glorieux de la nation, grâce à un programme culturel de vaste envergure fondé sur les personnalités iconiques du XIX siècle. Sous couvert d'un illusoire apolitisme de la musique, la radio et les concerts proclament partout le triomphe de la tradition romantique, celle de Beethoven, Wagner et Bruckner, jusque dans les pays conquis. L'œuvre des maîtres du passé doit stimuler les velléités nationalistes et démontrer la supériorité intellectuelle du peuple allemand.

2 La musique et la signification symbolique qu'elle véhicule doivent se propager dans toutes les sphères de la société. Les Jeunesses hitlériennes mettent à l'honneur les pratiques de groupes, chorales et instrumentales, et résonnent continuellement de chants de propagande martiaux. Outil d'humiliation également pour les victimes des camps de concentration, forcées de jouer pour divertir leurs bourreaux. Quant à la vie politique, elle est véritablement rythmée par l'art musical qui l'imprègne toute entière, comme en témoigne les propos de Philipp Bouhler :

Ce n'est pas un hasard si le Congrès du Parti commence chaque année par une exécution des Maîtres chanteurs. Qu'est-ce qui pourrait mieux convenir que cet immortel chef-d'œuvre de Richard Wagner? Il évoque la magie du vieux Nuremberg et signale, avec une éclatante puissance, le combat héroïque d'Adolf Hitler pour le peuple allemand. (Huynh, $2004: 59$ )

Dans le cinéma, la musique apparaît d'abord comme un moyen supplémentaire pour servir la propagande caractérisant l'ensemble des films produits sous le III ${ }^{e}$ Reich. Cinéma de genre et de studio comme le cinéma hollywoodien, la production nazie est essentiellement fictionnelle. La musique y est de qualité, son écriture est savante, en 
accord avec la tradition romantique. Tonale, elle fait montre d'une écriture contrapuntique expressive alliée à une certaine recherche harmonique, comme en témoignent par exemple les partitions de Münchhausen ou des mélos tels Immensee ou Romanze in Moll (Romance en mineur). On note également la présence importante de la musique chorale, ainsi que celle de la musique martiale, deux genres peu présents dans le classicisme hollywoodien. Mais ces variations n'empêchent pas une proximité évidente entre les deux styles musicaux, et, à plus forte raison, c'est l'organisation de la musique dans le cinéma du III ${ }^{\mathrm{e}}$ Reich qui rappelle sensiblement celle des films hollywoodiens. Il s'agit d'une musique cherchant à suivre l'action au plus près, dont la trame sonore évolutive et plastique renforce l'impact émotionnel de l'histoire, met en valeur ses points saillants. Le cinéma nazi étant un cinéma de propagande, la musique va nécessairement se mettre au service de l'idéologie, mais demeure secondaire en tant qu'elle est assujettie à l'image.

4 Il existe pourtant une production minoritaire non fictionnelle, plus particulièrement trois genres inconnus du cinéma américain. Le premier trouve son origine dans l'époque de Weimar, il se caractérise par un aspect documentaire ainsi qu'une dimension éducative et morale. Il s'agit du Kulturfilm, ou film culturel, sorte de voie médiane entre l'actualité filmée, documentaire, et le film de fiction. Il nous intéresse d'autant plus que la musique extradiégétique y est souvent très présente, malgré les prétentions documentaires du genre.

Le second trouve son origine dans la littérature du XIX ${ }^{\mathrm{e}}$ siècle. Le Heimatfilm est d'abord relatif à la culture allemande, mais, selon Éric Dufour, " même s'il est aussi culturel, il est d'abord et avant tout fondé biologiquement : c'est la nature comme valeur » (2014). Selon le concept de Blut und Boden (le sang et le sol) auquel le genre est associé, l'homme vivant en harmonie avec la nature, resté fidèle à ses origines, est considéré comme supérieur, ce qui fait du Heimatfilm un genre enclin à défendre des idées racistes et eugénistes.

6 Le troisième genre étroitement lié à la culture allemande puise ses sources dans la conception de la philosophie romantique incarnée par Schopenhauer, qui envisage le monde comme un tout organique, une unité indivisible. Richard Wagner s'est proclamé comme celui qui réaliserait par et dans l'art cette conception moniste, sous le concept d' art total, ambitionnant de rassembler et dépasser les différentes pratiques artistiques pour parvenir à l'universalité du drame. Il semble inévitable que de la fascination qu'exerce Wagner sur l'Allemagne naissent des tentatives pour prolonger sa pensée et tenter de réaliser grâce aux nouveaux moyens qu'offre le cinéma cette prophétique œuvre d'art de l'avenir.

7 Ainsi, le cinéma-art total relève davantage d'un principe formel que d'un genre à part entière. Le Tiefland de Leni Riefenstahl se rapproche de cette forme en tant qu'adaptation cinématographique de l'opéra du même nom, mais le film le plus fondateur est sans nul doute Ewiger Wald (Forêt éternelle), réalisé en 1936 par Hanns Springer et Rolf von Sonjevski-Jamrowski, «film de montage qui reste sans équivalent» (Cadars \& Courtade, 1972 : 56). À la croisée des genres spécifiquement allemands, il relève à la fois du Kulturfilm et du Heimatfilm.

8 L'ambition d'Ewiger Wald n'est rien moins que de retracer, en une suite de tableaux, l'histoire de l'Allemagne, depuis les temps immémoriaux jusqu'à l'avènement du III ${ }^{\mathrm{e}}$ Reich, en montrant comment le peuple se réalise en étroite communion avec la nature. 
Plus qu'un modèle social, la forêt, idéal métaphysique, inspire ses lois à la communauté humaine.

9 Les réalisateurs recourent à l'ensemble des moyens artistiques pour servir leur propos, mais la musique, composé par Wolfgang Zeller, affirme dès le départ son rôle prépondérant. Elle s'impose d'abord par son lyrisme, dès les premières images du film, en colorant les visions forestières esthétisées. La musique précède l'arrivée du verbe, incarné par la première injonction du narrateur, puis par le texte écrit, intertitre aux lettres gothiques présentant le thème du film. Il marque la fin de l'introduction et ouvre la voie au narrateur pour sa première véritable tirade rimée, sorte de poème épique qui servira de fil conducteur à l'œuvre dépourvue de dialogues. Ce dispositif évoque davantage celui d'un oratorio que celui d'un opéra, mais l'alliance de la musique, de l'image et de la poésie, au cœur de la problématique d'Ewiger Wald, révèle sa filiation avec la tradition opératique incarnée par Wagner.

10 L'homme et la nature sont des préoccupations wagnériennes de première importance. L'art se veut le reflet de l'homme vrai, non perverti par la société moderne et menant son existence selon une nécessité naturelle immanente. Trouvant dans la nature à la fois sujet et forme, l'œuvre d'art puise son inspiration dans la vie même. Elle est le meilleur moyen pour l'homme artiste d'exprimer sa nécessité la plus forte et la plus impérieuse, «se communiquer soi-même dans toute la plénitude de sa nature, à la communauté tout entière » (Wagner, 1982: 228). L'unique voie par laquelle l'homme parfait peut réaliser ce besoin premier est celle du drame, l'œuvre d'art de l'avenir.

11 Chaque genre artistique envisagé isolément possède ses limites expressives. «Danse, musique et poésie, séparément, sont bornées chacune à elle-même ; en se heurtant à ses limites, chacune d'elles se sent esclave si, parvenue à son point extrême, elle ne tend pas la main à l'autre genre d'art correspondant, avec un amour absolument reconnaissant " (Wagner, 1982 : 101). Ainsi, seule l'œuvre d'art totale, caractérisée par l'union de tous les arts, la ronde des trois soeurs, peut parvenir à exprimer de manière universelle « l'homme même, vivant et parfait » (Wagner, 1982 : 201). Le fondement du drame est d'être un art collectif, engendré par et pour le peuple. Le compositeur abandonne la conception romantique de l'artiste solitaire suivant sa propre voie : «L'esprit solitaire cherchant par l'art sa rédemption dans la nature, ne peut créer l'œuvre d'art de l'avenir: l'esprit collectif que la vie satisfait en est seul capable. » (Wagner, 1982 : 89)

Les opéras de la seconde période du compositeur (à partir de Tristan et Isolde) portent à leur perfection ses conceptions et travaux théoriques, dans une forme unitaire où le musicien-poète, qui écrit lui-même ses livrets, élabore une écriture musicale continue, sans cesse variée (Durchkomponiert), qui seconde le texte en suivant ses inflexions et son sens. Les œuvres de Wagner se construisent à partir d'un ensemble de Leitmotive, courts thèmes mélodiques ou harmoniques symbolisant des personnages, objets, affects, ou même idées métaphysiques. Ces Leitmotive assurent l'unité du discours musical, se mêlant à ses méandres ou s'affirmant avec force, se mélangeant entre eux, pour former un vaste réseau symbolique signifiant. Cette forme plastique trouve son aboutissement dans la Tétralogie, œuvre démiurgique en quatre volets retraçant la légende des Nibelungen, mythe médiéval fondateur de la culture germanique.

13 Cette description générale du style de Wagner permettra de comprendre en quoi il influence la forme d'Ewiger Wald. 


\section{Une ouverture opératique}

14 La première séquence d'Ewiger Wald consiste en un véritable hymne sans paroles à la nature, un portrait vivant et esthétique de la forêt. Les divers mouvements de caméra figurent un espace mouvant, bruissant, un vaste organisme. Le premier plan traverse latéralement la forêt avant d'évoluer jusqu'à la cime des arbres, mouvement ascendant qui figure la croissance, alors que le second, descendant, nous ramène en son cœur, sous la voute ombragée et mystérieuse. Ce double mouvement à la signification ambivalente, évoquant l'émancipation de l'homme et son ressourcement dans nature, à la fois dépassement et retour, sillonne l'ensemble de l'œuvre cinématographique.

Cette première séquence se veut la peinture du cycle des saisons, de la plénitude de l'été aux rigueurs de l'hiver, puis au renouveau du printemps, dans une appréhension circulaire du temps, directement inspirée des lois de la nature. Cette conception rappelle l'esprit du panthéisme, qui conçoit la nature comme expression du divin, les deux ne pouvant être dissociés, selon une appréhension de la religion comme immanente au monde. La musique suggère cette dimension religieuse de la forêt par l'utilisation d'une écriture vocale polyphonique évoquant le genre sacré, et parfois celle du choral luthérien. Celui-ci se caractérise par sa sobriété, et consiste en la mise en musique d'un texte religieux en langue populaire. Son écriture verticale, relativement homorythmique, la simplicité de ses intervalles et de ses rythmes le rend accessible à l'ensemble des fidèles. Toutes les voix chantent le texte en même temps, unies par l'harmonie, ce qui confère à ce genre une grande force symbolique.

Le thème du passage des saisons, c'est celui du cycle mort et renaissance, central dans le film, auquel la musique doit donner une image. Au ton lumineux initial de la majeur succède une modulation en ré mineur accompagnant les premières images de la dégénérescence liée à l'avènement de l'automne. C'est aussi la première fois que l'on entend une voix seule, féminine, dont les courbes mélodiques descendantes sont un figuralisme de la chute des feuilles.

Une nouvelle modulation, en mi mineur cette fois, accompagne un fondu enchaîné révélant la forêt au travers d'un halo de flocons de neige. L'image, caractérisée jusque-là par ses mouvements divers, se fige en une série de plans fixes figurant l'immobilité de la forêt, prise dans les nappes glacées de l'hiver. La musique, statique, se fait encore l'écho de cette immobilité : la voix de femme seule reparait avec une mélodie minimale brodant la note pivot initiale mi, accompagnée par une trame harmonique répétitive et les broderies des bois, tournant sur elles-mêmes. La basse elle-même, recto tono, est très peu mobile et renforce la sensation d'immobilité. 


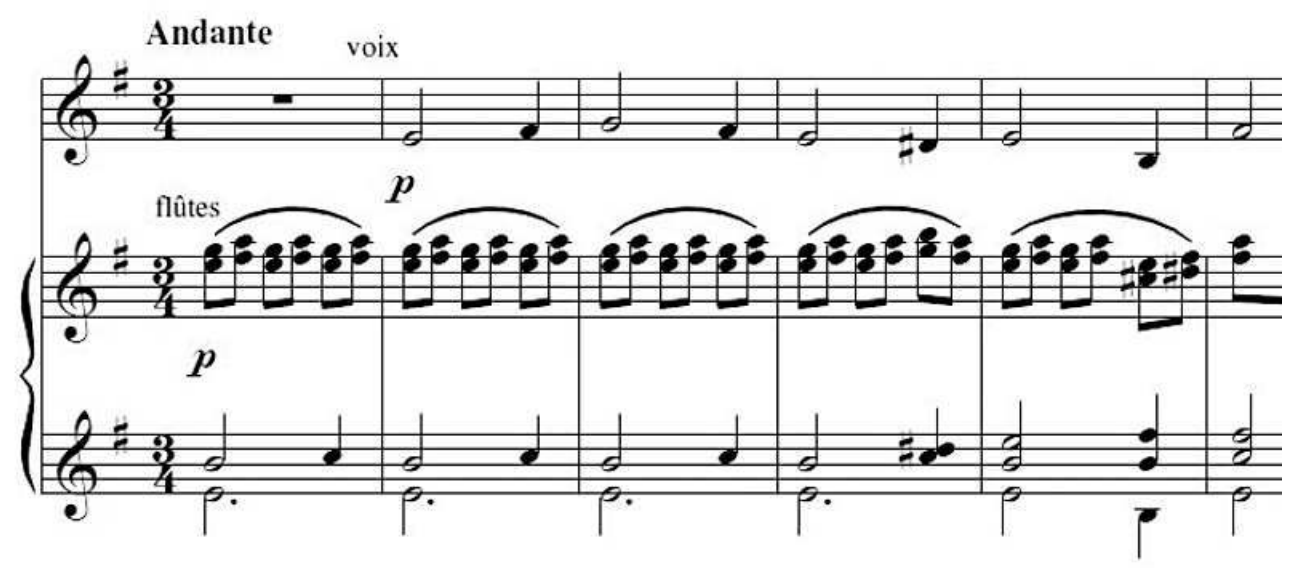

La nature s'anime à nouveau sous l'impulsion menaçante du vent, accompagnée par une musique chorale plus agitée. Les cuivres et timbales annoncent l'imminence de l'avalanche et la première sentence du narrateur, proférée avec véhémence et entrecoupée de grondements percussifs sinistres :

Ceux qui ne craignent pas la violence de l'hiver peuvent vaincre la mort.

19 Mais le printemps succède à l'hiver, la nature renait. Après les rayons du soleil perçant les nuages, les réalisateurs consacrent une suite de plans à l'eau, l'élément vital primordial qui, de plaques glacées fondant au soleil, se fait ruisseau, puis rivière, abreuvant la terre. La musique traduit le renouveau de la vie, joyeuse et dynamique, en recourant à une sorte de menuet, danse à trois temps chantée par le chœur dans une ambiance de liesse.

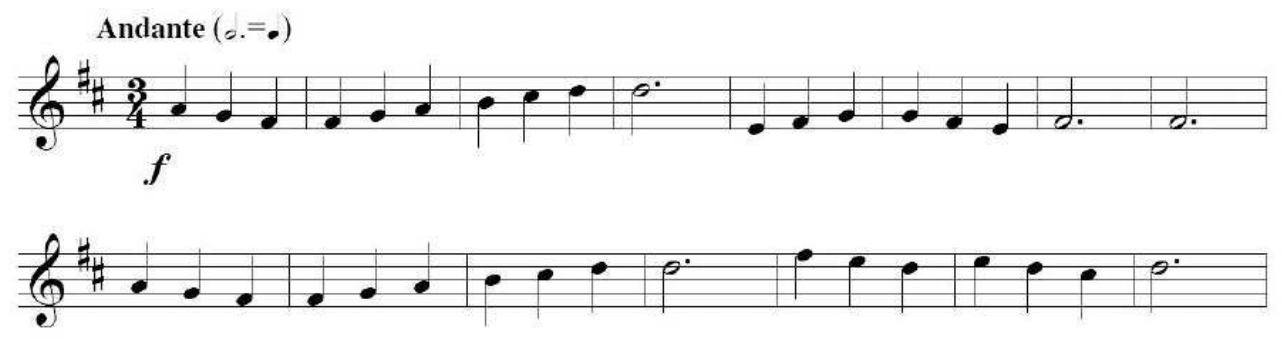

La séquence se conclut de manière théâtrale par un tutti orchestral retentissant en sol majeur, avec force cuivres et timbales, typique de la grandiloquence conclusive de nombreuses œuvres romantiques.

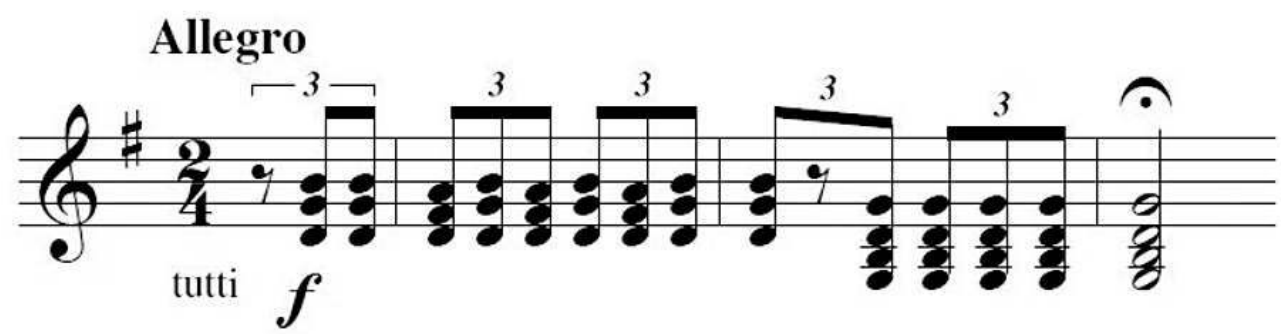

La construction de cette séquence introductive témoigne d'une forte influence opératique. En fait, elle consiste véritablement en une ouverture, ou prologue, terme désignant en musique la pièce instrumentale introduisant un opéra, sorte de musique narrative qui dépeint les grandes étapes de l'histoire racontée et évoque les thèmes principaux. Ainsi, l'ouverture fait entendre les motifs prégnants de l'œuvre, sortes de 
prémonitions que le spectateur reconnaîtra lors de leurs apparitions ultérieures, avant de se conclure souvent de manière brillante, affirmative.

On retrouve l'ensemble de ces caractéristiques dans le début d'Ewiger Wald. Hormis la seule sentence émise par le narrateur, la première séquence introduit le film avec les seuls moyens visuels et musicaux, sans le recours du verbe. De plus, tous les thèmes principaux du film y sont évoqués. Outre la figure de l'arbre et de la forêt, il y a celle de l'eau, ondoyante, symbole de vie, ou figée et immobile, neige et glace enveloppant toute chose dans un linceul mortuaire. On peut citer également l'image de la dualité entre émancipation de l'homme et retour à la nature telle que figurée par les premiers plans, et celle du cycle dégénérescence et régénération décrite par le passage des saisons et relayée par la musique, succession de mondes sonores contrastés jusqu'au triomphe de la lumière sur l'ombre. À celles-ci s'ajoutent le symbole de la violence, des lois implacables de la nature. C'est le vent mugissant, l'avalanche dévastatrice. Quant au thème de la communion des hommes, il est préfiguré par l'écriture chorale de la musique ainsi que l'utilisation de la mélodie de danse.

Tous ces symboles sont reliés et mis en valeur par cette musique ininterrompue, éminemment plastique, s'adaptant à l'image en variant autant ses timbres (chœur mixte, chœur d'homme, voix soliste, musique instrumentale seule...) que ses tonalités, ses dynamiques et ses caractères. Le style de Wolfgang Zeller est fidèle à la tradition wagnérienne, avec une musique très modulante aux harmonies recherchées, dans un discours Durchkomponiert.

Cependant, les Leitmotive ne sont pas musicaux mais bien visuels. On peut parler d'une transposition du sonore au visuel, où les symboles que montre le film jouent le même rôle que les motifs signifiants des opéras de Wagner. Le style du compositeur romantique est véritablement absorbé dans l'œuvre audio-visuelle, qui s'inspire de sa forme d'opéra pour organiser son discours et donner à voir les thèmes principaux avant de les présenter de manière plus univoque et explicite grâce au texte parlé. Si le premier niveau d'inspiration wagnérienne réside dans le principe formel du film, c'est-à-dire l'art total, le second niveau concerne l'organisation interne des images, déclinant les thèmes qui structureront le film et assureront son unité.

\section{La construction de l'idéologie}

Forêt éternelle, peuple éternel

Les arbres vivent comme toi et moi

Ils recherchent de l'espace comme toi et moi

Leur mort et leur naissance sont tissées ensemble dans le temps

La nation, comme la forêt, se dresse dans l'éternité.

Cette tirade est déclamée par Günther Hadank, qui est justement un acteur de théâtre, avec un souci esthétique évident. Pourtant, son ton prédicateur et impératif révèle une autre fonction que celle de poète. En vertu de la fonction didactique du Kulturfilm, le narrateur-poète est aussi professeur, ses mots constituent autant de leçons de morale et d'exhortations à l'action. Ils succèdent à un intertitre écrit en lettres gothiques qui dédie l'œuvre à tous les hommes appelant de leurs vœux « la construction du Reich éternel ».

Tout d'abord, comme le montrent Robert G. Lee et Sabine Wilke dans leur article « Forest as Volk: Ewiger Wald and the Religion of Nature in the Third Reich ", cet hymne à la nature prend la forme d'une sorte de néo-paganisme détourné, qui fait apparaître la forêt 
comme revêtant une dimension divine. Une atmosphère atemporelle, caractéristique de l'espace sacré, est engendrée par la présentation de scènes issues d'un passé immémorial, rituel païen, odyssée viking, Moyen Âge fantasmé, ambiance mystique renforcée par la présence continue de la musique. La dénonciation du christianisme, ennemi de cette religion de la nature, occupe une place importante dans le film. Le narrateur condamne l'arrivée des chrétiens qui, venus des régions d'un Sud pauvre en végétation, convoitent les forêts du Nord et s'installent en terre allemande, pendant qu'une succession de plans accompagnés d'une musique grave montrent la prolifération des crucifix investissant l'espace visuel. La musique elle-même participe à cette dénonciation par son recours à l'écriture du choral luthérien, symbole de la Réforme opposée à l'hégémonie de l'Église catholique, et de l'union retrouvée entre les croyants et leur foi.

Nombreux sont les effets cinématographiques appuyant ce propos. Après les croix, l'image décrit une morne et triste procession religieuse dans un couvent. Puis l'on voit l'une des nonnes tendre les mains vers la fenêtre de sa cellule avec une image de végétaux en surimpression, appel implorant de la nature. Cette scène est accompagnée d'un chant médiéval célébrant la forêt, qui contraste avec le style romantique dominant.

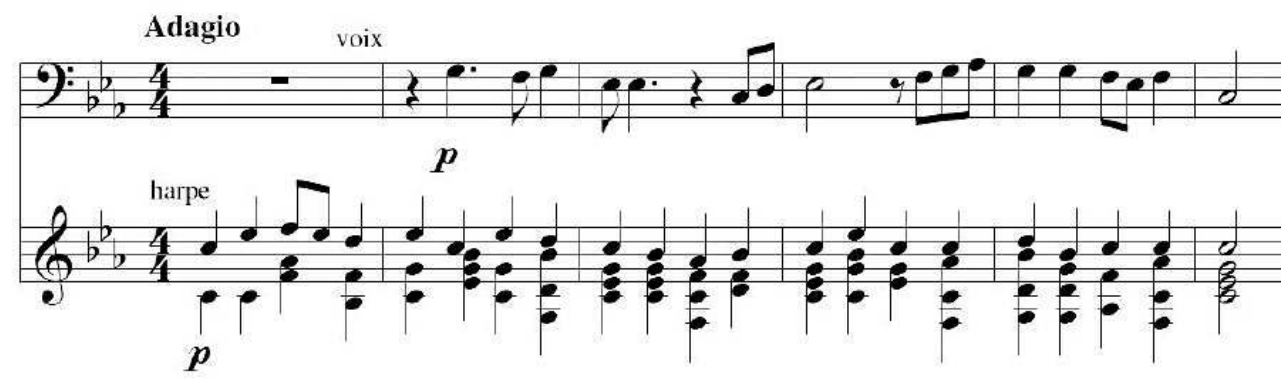

Cet hymne, dont la modalité et le timbre doux de la harpe participent au caractère archaïque, symbolise ici la pureté et l'expression vraie de l'homme naturel, non perverti par la société. Wagner lui-même n'aurait pas démenti cette conception, lui qui vantait cet "air populaire resté fidèle à lui-même, ayant conservé sa grâce intacte, la chanson étroitement confondue avec la poésie » $(1982: 128)$. Référence à l'amour courtois des troubadours ( $«$ je resterai ferme et loyal, oh reine, aie pitié de moi et laisse-moi te consacrer ma vie »), cet air crée une atmosphère de recueillement et de contemplation.

L'hommage rendu par Ewiger Wald à l'art gothique est également l'occasion de clamer le lien indéfectible entre spiritualité et nature. Le vitrail qui diffracte l'éclat du soleil en mille rayons ardents devient, par le fondu enchainé, une voute végétale piquée de ces mêmes taches de lumière. L'image de la plus haute tour d'une cathédrale se dissout par cette même figure de style, pour se reformer en un arbre majestueux.

La forêt est le fondement de la spiritualité humaine, elle est le modèle qui doit guider l'existence de chacun. La communauté doit réaliser son unité par et dans la forêt, en imitant ses lois.
Nous sommes originaires de la forêt
Nous vivons comme la forêt
Depuis la forêt nous bâtissons notre espace de vie
Nos âmes croissent comme les arbres
Pleines de vie, de joie, de drames, de questions.

Tout d'abord, la nature enseigne à l'homme que la mort est partie intégrante de la vie. C'est le thème du cycle mort et renaissance imprégnant toute l'œuvre, Leitmotiv dont on trouvera non moins que sept déclinaisons. Après le premier tableau, éloge de l'existence 
simple d'une communauté païenne en harmonie avec la nature, où le paganisme est représenté par une sorte de crucifix totémique orné d'une couronne végétale autour duquel danse une ronde de jeunes filles, le tableau suivant, consacré à la guerre des habitants de la forêt contre les envahisseurs romains, s'achève sur une cérémonie à la gloire des morts, accompagnée par une musique chorale digne du plus tragique des Requiem.

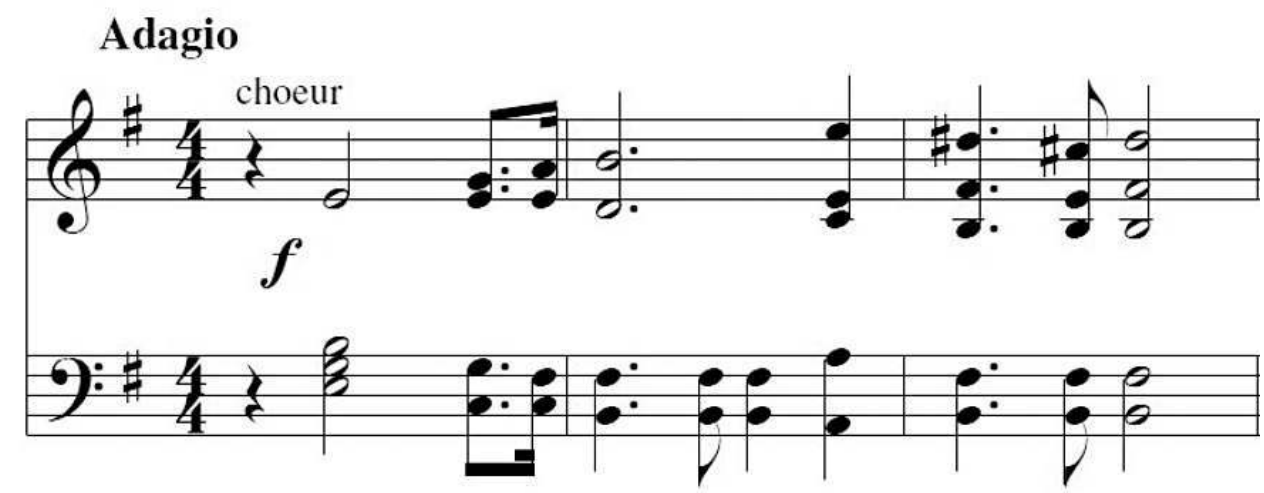

Le compositeur renforce la dramaturgie de la pièce par un discours très modulant aux harmonies expressives. L'exemple ci-dessus montre que, dès les premières mesures, Zeller varie les couleurs harmoniques avec la transformation du si mineur, modale et sombre, en un lumineux si majeur.

Cette scène permet aux cinéastes d'introduire un autre symbole phare dans leur œuvre, celui du feu. C'est l'unité, la fusion des âmes dans le recueillement et la contemplation, le feu vital originaire qui guide et rassemble les hommes. Si le corps se disperse en cendres et fumée, l'âme survit à travers la perpétuation de l'existence humaine. La scène culmine avec la surimpression d'images du peuple rendant hommage à ses disparus avec celle du «vieux signe runique en forme d'éclair» (Cadars \& Courtade, 1972: 57), référence à l'emblème de la SS, nimbé de flammes et brillant dans la nuit.

Les figures du Leitmotiv mort et renaissance seront toujours plus fortes à mesure que l'on s'achemine vers la résolution du film. Elles culmineront avec l'enchaînement entre un travelling latéral sur la désolation d'une immense forêt de croix mortuaires - soldats tombés au combat lors de la Première Guerre mondiale - et un gros plan sur un visage d'enfant. La jeunesse est la force vive et spontanée, l'expression naturelle de la nécessité supérieure de la nation allemande.

$\mathrm{Au}$ travers de cette idéologie glorifiant la mort, c'est l'idée de la guerre qui est en dernier recours visée. Si la mort est un bienfait, et si la forêt, mère du peuple, est menacée, alors l'homme ne doit pas craindre de se sacrifier pour sa défense. Tout le travail des cinéastes est de glorifier la guerre tout en faisant sans cesse apparaître le peuple germanique comme une victime forcée de se battre pour assurer sa survie.

La violence n'est pas condamnable car elle est au fondement des lois de la nature. Dans la scène de guerre contre l'envahisseur romain, séquence impressionnante au montage haletant, très resserré, la forêt devient elle-même une combattante épaulant les guerriers allemands. Des images d'éclairs illuminant le ciel, symbole de la force et du courroux de la nature, superposées à celles d'arbres agités par la bourrasque, alternent avec des images de lutte où les troncs dissimulent leurs défenseurs avant de s'abattre sur les oppresseurs, le tout sous-tendu par une musique symphonique épique. 

la victoire des paysans que culmine l'assimilation de l'homme et de la nature. Après que le peuple a replanté les arbres abattus, une métaphore très explicite fait alterner, via l'habituel fondu enchaîné, des plans montrant des rangées de militaires et des plans d'arbres s'élevant de plus en plus haut, ordonnés eux aussi en rangées régulières, devenant eux-mêmes des soldats en bataillons. On retrouve ici la dualité entre émancipation de l'homme et retour à la nature suggérée dans l'ouverture. Les hommes doivent s'inspirer des lois de la nature, mais ils façonnent une nouvelle forêt dont l'ordonnance est régie par les lois martiales proprement humaines. Dans cette dialectique trouble, où l'homme modifie la forêt censée dicter ses propres lois, apparaît la nature contradictoire de l'idéologie nazie. forme wagnérienne de l'opéra, en montrant comment les Leitmotive s'entrecroisent en vue d'un surcroît de signification. Les thèmes du cycle de la vie, la croissance, la communion, la violence, la guerre, la religion de la nature sont étroitement imbriqués en une structure complexe. Ils forment une trame sémantique qui unifie, en l'absence de narration, la succession rapide et sans cohérence apparente d'épisodes historiques, comme les Leitmotive wagnériens unifiaient le discours musical en constante évolution.

L'exemple du symbole de l'eau est représentatif. À la fin de la scène de lutte contre l'envahisseur romain, un soldat de l'empire chute dans une rivière et s'y effondre, avant de se noyer. L'étendard qu'il arborait chancelle quelques instants avant d'être englouti par les eaux. Un fondu enchaîné nous découvre cette même rivière apaisée, piquée de nénuphars, éclairée par les rayons du soleil. Il s'agit de la même eau, mais sa signification n'est plus la même: de délétère, associée à la guerre et la mort, elle est devenue vivifiante, saine. C'est cette dernière qui abreuve la nature renaissante (fin de l'ouverture) ainsi que l'humanité (le puits du premier tableau), ou permet aux hommes d'acheminer les troncs d'arbres pour bâtir leurs cités. Comme un Leitmotiv wagnérien, l'eau est changeante, mouvante, se pare de significations multiples en fonction du contexte. C'est aussi en termes de variation et d'interpénétration symbolique qu'il faut interpréter la superposition de l'insigne SS en flammes et du peuple assistant à l'oraison funèbre. La foule et le feu, deux symboles de communion, sont fusionnés grâce à la surimpression pour un regain de signification.

39 La finalité de ce vaste édifice symbolique, dans un contexte de propagande nazie, est sans équivoque la mise en images du fantasme de la supériorité raciale allemande.

\section{Ewiger Wald et l'idéologie wagnérienne}

On a montré que l'influence de Richard Wagner sur Ewiger Wald est de nature formelle, en tant qu'art total et que structure fondée sur la notion de Leitmotiv. Mais l'empreinte du compositeur romantique tient également au propos du film lui-même. Tout d'abord, celui-ci constitue une véritable exemplification des conceptions wagnériennes, selon lesquelles "[l'homme doit] se réunir de nouveau avec la nature dans l'œuvre d'art " (Wagner, 1982: 86). Cette union du peuple et de la forêt est bien le thème central de l'œuvre cinématographique. D'ailleurs, on y retrouve cette dialectique trouble du rapport entre les deux (l'homme est tantôt sujet et tantôt maître de la nature) déjà présente chez 
le compositeur, dans les ouvrages duquel on retrouve l'idée d'instrumentalisation de la nature par l'homme qui cherche à réaliser sa « volonté supérieure » (Wagner, 1982 : 180).

Selon Wagner, l'action dramatique est gouvernée par la figure d'un héros, dont l'acteurchanteur qui l'interprète doit faire rayonner l'existence édifiante. "Dans le drame, [l'homme] enrichit son caractère particulier en représentant un caractère individuel autre que lui-même, comme une personnalité humaine de caractère général. » (Wagner, 1982 : 229) C'est pourquoi « l'acteur artiste parfait est l'individu grandi jusqu'au caractère de l'espèce» (Wagner, 1982: 229). Or, Ewiger Wald s'organise tout entier autour de la figure de la forêt germanique, véritable héros selon la conception du compositeur, afin que son exemple imprègne et serve de modèle au spectateur fasciné. Sans que Wagner le théorise explicitement, il semble manifeste que le drame est de nature mythique. Exemplaire, fondateur d'une culture, le mythe est le type de récit le mieux à même de revêtir cette dimension intersubjective et de délivrer ses enseignements à tout un peuple. Hitler vouait un véritable culte à Wagner bien avant sa majorité (Huynh, 2004:32), et la lecture de ses ouvrages représente l'une des principales sources d'inspiration de Mein Kampf. Cet homme grandi jusqu'au caractère de l'espèce, c'est bien, dans l'idéologie nazie, le Führer lui-même, représentant de chair et de sang de l'esprit du peuple allemand.

La finalité d'Ewiger Wald, vers laquelle tend cette forme opératique, est d'ériger un mythe à la gloire de la forêt éternelle, à la fois modèle philosophique, éthique, mais aussi esthétique, en tant qu'exemple d'équilibre, de droiture, et fondement de l'art allemand. Mais au-delà du mythe de la forêt, c'est bien celui de la supériorité raciale que le film veut illustrer. Le dernier tableau, ronde populaire autour de «l'arbre traditionnel décoré pour le $1^{\text {er }}$ Mai » (Cadars \& Courtade, 1972: 57) exhibant la croix gammée, constitue l'avènement du nazisme, l'aboutissement de la longue épopée. L'image de la ronde des hommes tournant main dans la main autour de l'arbre-étendard est d'autant plus forte qu'elle reprend des éléments du début du film, selon le principe wagnérien : la ronde du premier tableau (le village païen), ainsi que le menuet entrainant de la fin de l'ouverture. Avant que le film ne s'achève sur un lent travelling latéral montrant l'immensité de la foule et de ses étendards nazis, symbole de l'unité réalisée et archétype de l'imagerie fasciste, le narrateur, au ton toujours plus enflammé et catégorique, prononce ces dernières paroles prophétiques :

L'étendard s'épanouit comme toi et moi

Sous le drapeau la nation m'appelle et t'appelle

Chante le nouveau cantique du temps!

Comme la forêt, la nation se dresse dans l'éternité.

Si la forêt subsiste symboliquement dans l'arbre-totem devenu démesurément grand, elle disparait de ce paysage urbain, révélant son véritable statut, simple prétexte idéologique. Dès le début du film, lorsque le narrateur déclame sa première tirade, l'image nous dévoile un arbre majestueux dont le tronc, scindé en deux à sa base, se rejoint au niveau des frondaisons pour ne former plus qu'un seul être. Ewiger Wald est un film sur la Volksgemeinschaft $^{1}$ (communauté du peuple), et l'avènement du Reich millénaire verra l'unité réalisée, le triomphe de la race aryenne, à la condition que le peuple, imitant la nature, lutte sans cesse pour sa survie.

Le travail de la forme cinématographique est donc de faire apparaître comme naturelle, émanant des lois même de la vie, une idéologie belliciste qui, bien qu'existant au XIX ${ }^{\mathrm{e}}$ siècle (pangermanisme), est en grande partie construite par le III ${ }^{e}$ Reich et élevée au rang de besoin vital. On voit nettement l'instrumentalisation de la nature à l'œuvre ici : le film 
plaque sur la figure de l'arbre et de la forêt une conception élaborée par l'homme seul. C'est ce que Lee et Wilke signifient lorsqu'ils parlent de « construction de l'imagination, projetée sur les arbres »(2005: 41). La forêt sert même à légitimer les convictions xénophobes et le programme eugéniste lorsqu'on la débarrasse des arbres morts ou tordus pour assurer son équilibre. « Coupez ce qui est malade et d'une race étrangère! » commande la voix impérieuse du narrateur.

La forêt telle que décrite dans Ewiger Wald est un mythe fabriqué par le nazisme. Comme chez Wagner, elle est ce héros dont tous doivent s'inspirer, modèle moral et philosophique. Mais le nazisme détourne la conception wagnérienne pour faire de ce héros un simple serviteur de l'idéologie.

\section{Conclusion}

Si Ewiger Wald relève de trois niveaux d'influence wagnérienne, ces niveaux sont intimement liés. Ce sont les conceptions philosophiques de Richard Wagner qui ont conditionné ses réalisations opératiques, et s'approprier sa forme musicale, c'est du même coup adopter toute une Weltanschauung (conception du monde) qui influe sur la création. Cette citation montre à quel point l'œuvre d'art de l'avenir est porteuse d'un pesant sous-texte idéologique :

La grande œuvre d'art totale qui devra englober tous les genres de l'art pour exploiter en quelque sorte chacun de ces genres comme moyen, pour l'annihiler en faveur du résultat d'ensemble de tous les genres, c'est-à-dire pour obtenir la représentation absolue, directe, de la nature humaine accomplie, - il ne reconnaît pas cette œuvre d'art totale comme l'acte volontairement possible d'un seul, mais comme l'œuvre collective nécessairement supposable des hommes de l'avenir. (Wagner, $1982: 87$ )

Chaque genre d'art se donne tout entier dans le drame en renonçant à son individualité. "Ce n'est que si chacun disparait en tant qu'art isolé, qu'ils deviendront tous capables de créer l'œuvre d'art parfaite; cette absorption en ce sens sera déjà d'elle-même cette œuvre d'art, et leur mort sera immédiatement la vie de cette œuvre d'art. " (Wagner, 1982 : 177) Cette idéologie, c'est bien celle du sacrifice personnel en faveur de l'ensemble, où l'individualité ne trouve pas sens en elle-même mais seulement en tant que partie d'un tout plus vaste, d'une réalité supérieure conçue comme transcendante. Une nouvelle fois, il semble que l'on puisse concevoir Ewiger Wald comme un détournement de cette idéologie, une forme de transposition de la conception esthétique de Wagner en une conception politique. Cette analogie est rendue possible par la proximité entre l'œuvre d'art totale wagnérienne et la conception nazie du III Reich, à la fois œuvre d'art et une finalité en soi. La conception glorificatrice de la mort, conception cyclique qui la fait apparaître comme une voie vers la renaissance et la croissance, vers le triomphe inextricablement mêlé de la forêt et du peuple allemand, autorise à lire l'idéologie qui imprègne le film à l'aune de celle de Wagner. Cet esprit de sacrifice individuel - «jouissance esthétique de premier ordre » (Kreimeier, 1994: 372) selon les termes de Walter Benjamin - est présenté comme la voie nécessaire vers la réalisation achevée du peuple allemand, porté à sa perfection dans le III ${ }^{\mathrm{e}}$ Reich.

Si l'idéologie nazie semble à ce point inspirée de l'œuvre littéraire de Wagner, c'est-à-dire d'une œuvre de philosophie esthétique, c'est aussi parce que le discours idéologique national-socialiste revêt la forme d'un discours proprement artistique. En effet, ce discours repose moins sur un raisonnement rationnel, émaillé d'arguments visant à 
démontrer la véracité d'une thèse, que sur un champ lexical de nature symbolique, un ensemble de mots porteurs d'images et d'affections, tenant davantage de la poésie. Walter Benjamin avait lui-même proposé la notion d'« esthétisation de la politique » dans son ouvrage L'Euvre d'art à l'époque de sa reproductibilité technique. Les discours politiques du III e Reich s'apparentent à des poèmes cherchant à susciter des émotions grâce à un ensemble de termes symboliques (drapeau, nation, peuple, fidélité, etc.) dans lesquels se retrouve toute la communauté, exactement dans l'esprit des tirades du narrateur d'Ewiger Wald.

49 Conception esthétique de la race aryenne, appréhension du III Reich comme l'œuvre d'art finale et parfaite, essence artistique de la forme de pensée nazie, tout concourt à la définition de l'idéologie national-socialiste, selon les termes de Philippe Lacoue-Labarthe et Jean-Luc Nancy, comme « la construction, la formation et la production du peuple allemand dans, par et comme une œuvre d'art » (1991: 49).

Klaus Kreimeier illustre cette tendance à l'esthétisation avec la mise en scène de la visite de Mussolini à Berlin, en septembre 1937, «manœuvre d'illusion unique en son genre, une machination esthético-politique qui visait à enivrer et à désactiver la réalité » (1994: 372). Voilà le pouvoir de l'art total wagnérien, dont Ewiger Wald évoque à tous égards un prolongement, la réalisation la plus achevée. Il s'appuie sur l'ensemble des possibilités expressives pour submerger le public de stimulations sensorielles. Les arts réunis donnent à la figure de la forêt une aura mythique qui enveloppe le spectateur et l'amène à adhérer plus sûrement à l'idéologie. Dès 1929, Alfred Rosenberg, penseur autoproclamé du Reich à venir, déclarait au congrès du Parti que «transmettre par des symboles suggestifs - couleurs, signes, sons - un être collectif, représenter un mythe vital» (Huynh, 2004: 179), était l'une des principales fonctions du NSDAP. S'adressant aux affects plutôt qu'à l'intellect, l'art total, de nature immersive, peut plonger le spectateur dans l'histoire pour étouffer tout sens critique. Le principe de déclinaison à l'infini des mêmes thèmes, caractéristique de la propagande nazie, se retrouve dans cette forme en Leitmotive fondée sur la répétition, dont le flot ininterrompu martèle l'idéologie. Voilà l'instrumentalisation du modèle wagnérien à l'œuvre, arme de propagande idéale.

51 Ewiger Wald est financé par la N.S. Kulturgemeinde (Communauté culturelle nationalesocialiste), «organisation chargée de réorganiser l'ensemble de la scène artistique et culturelle du III Reich en la recentrant sur l'idéologie nationaliste » (Lee \& Wilke, 2005 : 33). Il exemplifie l'une des principales caractéristiques du cinéma nazi : l'art de distordre la réalité pour faire apparaitre le peuple allemand comme une victime. Pseudohistorique, cette "allégorie de l'histoire et de la vie ${ }^{2}$ ", non narrative, n'en est pas moins téléologique. Chaque son, chaque mot, chaque image n'a qu'un but, présenter l'avènement du $\mathrm{III}^{\mathrm{e}}$ Reich comme une nécessité naturelle, un aboutissement logique définitif. Ewiger Wald prétend filmer la fin de l'Histoire.

Présenté comme un documentaire, le film annihile toute possibilité objective et factuelle par son extrême esthétisation, comme l'atteste l'expressivité de la caméra et son large panel de figures de style visuelles. Ewiger Wald est ce spectacle « total et fusionnel, où le spectateur, subjugué, abasourdi, enivré et transporté abdiquait tout jugement » (Clair, 2003 : 150). Mais dans l'ensemble des arts, c'est bien la musique, l'art le plus populaire, expressément désigné par Wagner comme la langue du cœur, le mieux à même de parler au sentiment plutôt qu'à la raison par son pouvoir suggestif, voire manipulateur :

Le lyrisme d'Orphée n'aurait certes pas pu forcer les bêtes féroces à une attitude

silencieuse et calme, si le chanteur n'avait fait que leur donner à lire des vers 
imprimés : il fallut que s'imposât tout d'abord à leurs oreilles la voix sonore du cœur, [...], de telle sorte qu'elles ne vissent plus nécessairement en cet homme seulement un objectif pour leur estomac, bon à être mangé, mais encore un sujet digne d'être écouté et vu, avant qu'elles devinssent capables de faire attention à ses sentences morales. (Wagner, $1982: 150)$ d'un « désir inassouvi, illimité » (135), charge affective primordiale qui doit être mise en forme et explicitée par le verbe. En un sens, la musique est première car elle représente des affects et sensations purs auxquels l'image doit donner une forme. En tant qu'art non figuratif, la musique symphonique tonale, par son lyrisme et sa force affirmative, peut faire triompher n'importe quelle idée. La puissance expressive de l'écriture wagnérienne, avec les ouvertures de Rienzi et des Maitres chanteurs de Nuremberg, était massivement utilisée lors des meetings politiques pour transfigurer les discours d'Hitler, décupler leur puissance affective. Plus qu'une arme de propagande, la musique apparaît pour le III $^{\mathrm{e}}$ Reich comme un idéal. Si le nazisme a besoin de la rhétorique verbale, par lui le langage est vidé de sa substance, rendu inintelligible, il ne sert pas à construire une réflexion mais à susciter des émotions. Or, la musique est l'art irrationnel par excellence qui, par le concours figuratif du langage et de l'image, peut seul susciter un mode d'adhésion profond et inconditionnel, contournant l'intellect pour prendre possession de l'affectivité.

En opérant cette union entre les trois genres artistique, Ewiger Wald fait vivre et prolonge la conception wagnérienne de l'art total grâce aux possibilités nouvelles du cinéma. Mais il apparaît que la préoccupation esthétique du film est aussi politique, l'art total étant la forme artistique idéale pour ancrer une idéologie dans l'esprit des spectateurs. Si l'on peut parler de détournement de la philosophie wagnérienne à des fins de propagande, il est difficile d'affirmer dans quelle mesure Ewiger Wald constitue une perversion de la pensée du compositeur, celle-ci renfermant déjà toute une idéologie sous-jacente que saura exploiter le national-socialisme.

\section{BIBLIOGRAPHIE}

CADARs Pierre et CoURTADE Francis (1972), Histoire du cinéma nazi, Paris : Éric Losfeld.

CLAIR Jean (2003), Du Surréalisme considéré dans ses rapports au totalitarisme et aux tables tournantes, Paris : Mille et Une Nuits.

DUFOUR Éric (2014), La Représentation du mal dans le cinéma allemand, Paris : Armand Colin.

HuYnh Pascal (dir.) (2004), Le III Reich et la Musique, 8 octobre-9 janvier 2005, Musée de la musique, catalogue d'exposition, Musée de la musique / Fayard.

KREIMEIER Klaus (1994), Une Histoire du cinéma allemand : la Ufa, Paris : Flammarion.

LEE Robert G. \& WILKE Sabine (2005), « Forest as Volk: Ewiger Wald and the Religion of Nature in the Third Reich », Journal of Social and Ecological Boundaries, printemps 2005, 1(1), 21-46, en ligne sur <www.tamiu.edu/coas/jseb/11files/ForestAsVolk.pdf>. 
LACOUE-LABARTHE Philippe et NANCY Jean-Luc (1991), Le Mythe nazi, La Tour-d'Aigues : L'Aube.

WAGNER Richard (1982), L'Euvre d'art de l'avenir, Plan-de-la-Tour : Éditions d'Aujourd'hui.

\section{NOTES}

1. Cette notion est tout d'abord illustrée dans le film par la surprésence de la musique chorale. Le chant en groupe est le meilleur moyen pour éprouver à la fois dans son corps (c'est l'activité musicale la plus directement physique, le corps est lui-même instrument) et son esprit, par le rythme et l'harmonie, la communion avec les autres.

2. Il s'agit du complément du titre du film Ewiger Wald.

\section{RÉSUMÉS}

Ewiger Wald, à la croisée des genres spécifiquement germaniques, relève à la fois du Kulturfilm, genre à prétention documentaire possédant une dimension éducative et morale, et du Heimatfilm, explorant le rapport entre l'homme et la nature, et possédant des connotations raciales. Mais surtout, le film relève d'une conception totalisatrice caractéristique de la tradition musicale allemande et incarnée par la figure tutélaire de Wagner. Ewiger Wald adapte la forme sonore des opéras wagnériens au domaine visuel en construisant la forme cinématographique à partir de divers symboles signifiants, l'eau, le feu, le cycle de la renaissance succédant à la mort, dont l'insistance renforce l'idéologie sous-jacente. La forêt est représentée comme un modèle dont les hommes doivent adapter les lois à leur propre vie. C'est une manière de rendre légitime l'idéologie raciale et belliciste en projetant sur la forêt les principes du nazisme. L'importance accordée à la dégénérescence, ainsi qu'à la lutte pour la survie, dans le mode d'existence de la forêt, permet de justifier le sacrifice pour la patrie ainsi que la théorie de l'espace vital. Ewiger Wald s'inspire également de la pensée de Richard Wagner : la fusion de l'homme et de la nature dans l'œuvre d'art, l'unification des arts où chacun abandonne son individualité pour se fondre dans un tout qui le dépasse. Au-delà du film, l'idéologie nazie veut procéder ainsi avec le peuple lui-même, par la fusion des âmes dans le Führerprinzip. L'appareil étatique est conçu comme un vaste spectacle qui prend en charge l'affectivité. Les discours politiques, vides de contenus argumentatifs, excitent les représentations mentales du public par des termes symboliques, devenant eux-mêmes musique. L'entendement, inondé de stimulations sensorielles, tend à abdiquer tout jugement.

Ewiger Wald, zugleich Heimat- und Kulturfilm, bezieht sich auf Traditionen des deutschen Films, vor allem aber auf das auf Wagner zurückzuführende Gesamtkunstwerk. Im Film wie in der Wagnerschen Oper wirken Musik, Bild und dichterisches Wort zusammen. Die filmische Umsetzung des Wagnerschen Ansatzes verwendet Symbole wie Wasser, Feuer, Tod und Wiedergeburt. Den Gesetzen des Waldes unterwerfen sich die Menschen, der Wald gerät somit zur Projektionsfläche der NS-Weltanschauung. Lebensraum und Lebenskampf stehen im Mittelpunkt sowie der Krieg gegen die Entartung, der den Tod fürs Vaterland rechtfertigt. Im Film bedingt die Musik Affekte, die den Zuschauer auf die NS-Ideologie einstimmen. Ewiger Wald bezieht sich auch auf das Denken Richard Wagners, auf das Einswerden von Mensch und Natur 
im Kunstwerk, auf das Einswerden aller Künste, auf das Aufgehen des Einzelnen im Ganzen, im Führerprinzip. Politik gerät zum emotionsgeladenen Spektakel, politische Reden wollen nicht überzeugen sondern das Publik vermittels einer symbolischen gleichsam musikalischen Sprache politisch aufreizen. Der Mensch wird seines Verstandes beraubt und auf seine stimulierten Sinne reduziert.

INDEX

Mots-clés : cinéma nazi, idéologie nazie, film de propagande, totalitarisme, instrumentalisation, Histoire, peuple allemand, forêt, nature, musique, art total, leitmotiv

Schlüsselwörter : Film im Dritten Reich, Nationalsozialismus, Propagandafilm, Totalitarismus, Instrumentalisierung, Geschichte, deutsches Volk, Wald 